\title{
Patient Perceptions of Psoriatic Arthritis Management and Communication with Physicians in Australia: Results from a Patient Survey
}

\author{
Irwin Lim · Pascal Richette $\cdot$ Ruben Queiro-Silva $\cdot$ Jade Moser • \\ Joseph C. Cappelleri · Ho Yin Ng (D) · David Witcombe
}

Received: December 17, 2020 / Accepted: February 25, 2021 / Published online: March 24, 2021

(c) The Author(s) 2021

\begin{abstract}
Introduction: The objective of this report was to evaluate perceptions of psoriatic arthritis (PsA) treatment and satisfaction with healthcare professional (HCP) communication among patients with PsA in Australia, compared with overall global perceptions.

Methods: Data were collected via a global and country-specific survey (The Harris Poll; November 2, 2017-March 12, 2018). Eligible patients were $\geq 18$ years old, had been diagnosed with PsA $>1$ year prior, had seen a
\end{abstract}

Supplementary Information The online version contains supplementary material available at https:// doi.org/10.1007/s40744-021-00297-w.

I. Lim

BJC Health, Sydney, NSW, Australia

P. Richette

Lariboisière Hospital, University of Paris 7,

Lariboisière, Paris, France

R. Queiro-Silva

Rheumatology Division, HUCA, Oviedo, Spain

J. Moser

The Harris Poll, Rochester, NY, USA

J. C. Cappelleri

Pfizer Inc, Groton, CT, USA

H. Y. Ng (殴 · D. Witcombe

Pfizer Australia, Sydney, NSW, Australia

e-mail: patrick.ng1@pfizer.com rheumatologist or dermatologist within the past 12 months, and had previously received $\geq 1$ conventional synthetic or biologic diseasemodifying antirheumatic drug. Data reported by patients included baseline demographics, overall health, time since PsA diagnosis, PsA severity, satisfaction with current PsA medication and management, and experiences regarding communication with their HCP. Descriptive statistics were obtained.

Results: Most patients in Australia were very or somewhat satisfied with their PsA medication, and reported always or often taking their medication exactly as directed by their HCP. However, the majority still experienced symptoms, reported their overall health as poor or fair, and would change something about their PsA medication. While the majority of patients in Australia were satisfied with the communication with their HCP, most would prefer increased communication but some felt that asking too many questions would affect the quality of their care. Perceptions in Australia were similar to global perceptions.

Conclusions: Although most patients with PsA in Australia were satisfied with their disease management and communication with their HCP, many still experienced symptoms, would change something about their PsA medication, and would prefer increased communication with their HCP. 


\section{PLAIN LANGUAGE SUMMARY}

Psoriatic arthritis (PsA) can cause tender and swollen joints. If not treated properly, the joint damage can get worse, until patients struggle to cope with everyday tasks. Patients and their doctors need to communicate well to successfully manage PsA. We used an online survey to ask patients in Australia how they feel about their PsA medication and the way they communicate with their doctor. These patients were adults who had had PsA for more than 1 year, had seen a specialist doctor in the past year, and had taken one or more prescription PsA medications. A total of 152 patients in Australia completed the survey. Most patients were very or somewhat satisfied with the PsA medication they were taking, and most always or often took it exactly as their doctor told them to. However, almost all patients still had symptoms, most said their overall health was poor or fair, and most would like to change something about their medication. While most patients were satisfied with the communication with their doctor about PsA, most wished they talked more with their doctor about their PsA and treatment goals, but some felt that asking too many questions would harm their quality of care. Patients in Australia had similar answers to patients who answered the survey in other countries. Although the survey was limited by the number of patients who responded, and whether patients answered questions properly, it suggests that patients and doctors need to communicate more closely to improve PsA management.

Keywords: Disease-modifying antirheumatic drug; Physician-patient relations; Psoriatic arthritis; Rheumatic diseases

\section{Key Summary Points}

\section{Why carry out this study?}

Psoriatic arthritis (PsA) can significantly impact quality of life.

Effective patient-healthcare professional (HCP) communication has been identified as a key factor for improving the management of PsA.

We evaluated perceptions of PsA treatment and satisfaction with HCP communication among patients with PsA in Australia, compared with overall global perceptions.

\section{What was learned from the study?}

Our survey data revealed that patients with PsA in Australia were satisfied with their disease management and communication with their HCP; however, many still experienced symptoms, would change something about their PsA medication, and would prefer increased communication with their HCP.

Our data highlight a need for improved medication management and increased HCP communication among patients with PsA in Australia.

\section{DIGITAL FEATURES}

This article is published with digital features, including a summary slide, to facilitate understanding of the article. To view digital features for this article go to https://doi.org/10.6084/ m9.figshare.14075477.

\section{INTRODUCTION}

Psoriatic arthritis (PsA) is an inflammatory disease characterized by peripheral arthritis, enthesitis, dactylitis, spondylitis, and psoriatic 
skin and nail disease [1, 2]. PsA is associated with tender, swollen joints and functional impairment, and if left untreated can result in progressive structural damage of affected joints [1]. A meta-analysis of pooled data from 266 studies estimated the global prevalence of PsA in patients with psoriasis to be $19.7 \%$ [3]. Patients with PsA have increased functional disability and decreased quality of life, compared with patients with psoriasis [4] and the general population [5]. Additionally, comorbidities (e.g., hypertension, obesity, and type 2 diabetes mellitus) are more prevalent in patients with PsA compared with patients with psoriasis [6].

For patients with PsA, achieving clinical remission or low disease activity (LDA) requires an early diagnosis by a physician, early commencement of treatment to delay joint damage, and appropriate monitoring of clinical response to treatment [7-9]. Accordingly, effective patient-healthcare professional (HCP) communication has been identified as a key factor for improving the management of PsA [10].

Shared decision-making between a patient and their HCP is included in international management guidelines for PsA, and is a key driver for a treat-to-target approach [11]. However, a patient's perception of disease remission or disease activity may differ from the perception of their HCP, and communication between a patient and their HCP is not always optimal [10]. A global and country-specific survey was conducted by The Harris Poll on behalf of Pfizer Inc (PsA narrative global patient survey) to determine the impact of PsA on daily life from the patient's perspective [12]. Here, we present data from The Harris Poll on perceptions of PsA treatment and satisfaction with HCP communication among patients in Australia with PsA. Global patient perceptions (from eight countries, including Australia) are also presented for context.

\section{METHODS}

\section{Study Design and Patients}

The Harris Poll conducted a patient-based online survey in Australia, Brazil, Canada,
France, Spain, Taiwan, the UK, and the USA from November 2, 2017 to March 12, 2018. Patients were recruited from online market research panels made up of members who agreed to participate in this type of research. Prospective respondents completed an initial survey screener; once identified as eligible participants, patients were required to provide consent in order to continue to the core survey content.

Each country had a custom set of demographic questions, followed by a dynamic number of questions, which varied depending on patient responses. In Australia, respondents were presented with a range of 37-57 questions (9 demographic and 28-48 custom questions). No qualitative questions were asked in this survey.

Eligible patients were $\geq 18$ years old with a self-reported diagnosis of PsA for $>1$ year prior to participation in the survey. Furthermore, patients were required to have visited a rheumatologist or dermatologist within the past 12 months and had previously received $\geq 1$ conventional synthetic or biologic diseasemodifying antirheumatic drug (DMARD) for PsA.

Estimated qualification rates were determined based on the approximate total number of patients who entered the survey versus the final qualified completers.

\section{Endpoints}

Data reported by patients included baseline demographics, overall health, time since PsA diagnosis, PsA severity, satisfaction with current PsA medication and management, and their experiences regarding communication with their HCP.

Data pertaining to communication with their HCPs were reported separately for patients mostly managed by a rheumatologist and patients mostly managed by a dermatologist.

\section{Statistical Analysis}

This analysis compared survey data from patients in Australia with data from the global patient survey. Data were evaluated using descriptive statistics. Reported $N$ values are 
unweighted, i.e., they reflect the actual number of patients who completed the survey. Reported percentage values for Australia are based on weighted data; weighted data were adjusted to account for the relative size of each country's population (as opposed to the number of respondents for each country) within the total eight-country global population [13].

\section{Ethics Approval}

Patients were recruited from online market research panels made up of members who agreed to participate in this type of research. Qualified respondents provided informed consent to complete the research. The surveys were non-interventional and were not conducted as a clinical study. All respondents agreed to participate, however, due to the nature of the study, ethics approval was not required.

\section{RESULTS}

\section{Patients}

In total, 152 patients in Australia with PsA were included in this analysis (raw unweighted patient population). The total estimated qualification rate for patients in Australia was 32\% (152/480). After weighted data were adjusted to account for the relative size of the country's adult population within the total adult population for the global survey, the sample size for patients in Australia was 39. The global population consisted of 1286 patients, and these data are presented in detail elsewhere [12]. Patient demographics and disease characteristics for patients in Australia and the global patient population are described in the electronic supplementary material (Table S1).

In Australia, the majority of patients were male and employed. The mean (standard deviation) age and time since their PsA diagnosis was 45.7 (13.7) and 8.5 (8.2) years, respectively. Most patients reported moderate PsA severity (61\%) and fair overall current health (55\%). The majority of patients (69\%) were currently taking biologic or oral DMARDs, or both, for their PsA (see Table $\mathrm{S} 1$ in the electronic supplementary material).

Global data are presented in the electronic supplementary material (Table S1) and described in detail elsewhere [12]. Demographic characteristics were generally comparable with patients in Australia, except for a higher proportion of patients with a current prescription of only biologic DMARDs in the global population (38\% [global] vs. 19\% [Australia]). Most patients in Australia (76\%) and most global patients $(76 \%)$ considered a rheumatologist to be the primary HCP responsible for managing their PsA (see Table S1 in the electronic supplementary material).

\section{Medication Satisfaction}

In Australia, three-quarters of patients (75\%) reported that they were very or somewhat satisfied with their current PsA medication regimen (Table 1). Similarly, most global patients $(84 \%)$ were very or somewhat satisfied with their current PsA medication. In Australia, the most commonly reported reasons for stopping PsA medication included: side effects difficult to manage, and no improvement with/worsening joint symptoms (Table 1). Globally, the most frequent reasons for halting PsA medication were concerns with potential serious side effects and persistent or worsening fatigue.

Of the patients in Australia, 55\% reported always taking their PsA medication exactly as directed by their HCP; however, 90\% still experienced musculoskeletal symptoms (Table 2). Additionally, most patients in Australia (93\%) expressed a desire to change aspects of their current PsA medication regimen. The most frequently reported aspects to change about their current medication included: taking fewer medications (43\%), improving the efficacy of medications to relieve musculoskeletal symptoms $(42 \%)$, and reducing the number or severity of side effects (37\%) (Table 2). Globally, $65 \%$ of respondents reported always taking their PsA medication as directed by their HCP; however, 91\% still experienced musculoskeletal symptoms (Table 2). Moreover, 93\% of global respondents expressed a desire to change 
Table 1 Patient satisfaction with PsA medication and rationale for discontinuation

\begin{tabular}{|c|c|c|}
\hline & \multicolumn{2}{|c|}{$\begin{array}{l}\text { Respondents currently taking prescribed medication } \\
\text { for PsA }\end{array}$} \\
\hline & Australia & Global \\
\hline Unweighted population, $N$ & 149 & 1270 \\
\hline Weighted base, $N$ & 38 & 1270 \\
\hline \multicolumn{3}{|l|}{ Satisfaction with PsA medication regimen, $n(\%)^{\mathrm{a}}$} \\
\hline Very satisfied & $9(23)$ & $438(35)$ \\
\hline Somewhat satisfied & $20(52)$ & $624(49)$ \\
\hline Somewhat dissatisfied & $8(22)$ & $138(11)$ \\
\hline Very dissatisfied & $1(3)$ & $70(6)$ \\
\hline Reasons for stopping PsA medication(s), $N^{\mathrm{b}}$ & $49^{c}$ & 278 \\
\hline Side effects difficult to manage, $n(\%)$ & $5(43)$ & $66(25)$ \\
\hline No improvement with/worsening joint symptoms, $n$ (\%) & $5(41)$ & $68(26)$ \\
\hline Persistent or worsening pain, $n(\%)$ & $5(39)$ & $55(21)$ \\
\hline Concerns with potential serious side effects, $n(\%)$ & $4(35)$ & $79(30)$ \\
\hline Persistent or worsening fatigue, $n(\%)$ & $4(33)$ & $69(27)$ \\
\hline Persistent or worsening enthesitis, $n$ (\%) & $4(33)$ & $59(23)$ \\
\hline
\end{tabular}

The unweighted population reflects the total number of patients who completed the survey and were currently taking prescribed medication for PsA; all reported percentages are calculated based on the weighted population as the denominator. Percentages might not exactly match those derived by manual calculation, due to weighting and/or computer rounding. All $n$ numbers and percentages are shown to the nearest integer

$N$ unweighted total population of respondents, $n$ number of respondents within given category (based on weighted population), Ps $A$ psoriatic arthritis

a Reported in response to the question: 'You indicated that you are currently taking prescription medication(s) for PsA. Overall, how satisfied are you with your current PsA medication regimen?'

b In patients who stopped taking medications in the past 2 years, reported in response to the question: 'Thinking of the most recent time you stopped taking your PsA medication(s), which of the following, if any, are reasons why you stopped? Please select all that apply'

c Very small base size $(n<50)$; results should be interpreted as directional only

aspects of their current PsA medication regimen, and the most commonly reported aspects to change about their current medication were similar to those reported by the patients in Australia (Table 2).

\section{Satisfaction with HCP Communication}

The large majority of patients in Australia reported that they were very or somewhat satisfied with the communication with their rheumatologist $(85 \%)$ or dermatologist $(94 \%)$. Similarly, most global patients were very or somewhat satisfied with the communication with their HCP (rheumatologist, 89\%; dermatologist, $88 \%$ ).

With regards to communication, most patients in Australia, and most global patients, felt that their HCP handled the following aspects very or somewhat well: explaining treatment options in understandable words, explaining the disease in understandable words, 
Table 2 Adherence to, and effectiveness of, PsA medication

\begin{tabular}{|c|c|c|}
\hline & \multicolumn{2}{|c|}{$\begin{array}{l}\text { Respondents currently taking } \\
\text { prescribed medication for PsA }\end{array}$} \\
\hline & Australia & Global \\
\hline Unweighted population, $N$ & 149 & 1270 \\
\hline Weighted base, $N$ & 38 & 1270 \\
\hline \multicolumn{3}{|l|}{ Takes PsA medication exactly as directed by $\mathrm{HCP}, n(\%)^{\mathrm{a}}$} \\
\hline Always & $21(55)$ & $824(65)$ \\
\hline Often & $11(30)$ & $328(26)$ \\
\hline Sometimes & $5(13)$ & $79(6)$ \\
\hline Rarely & $1(1)$ & $33(3)$ \\
\hline Never & $\mathrm{b}(1)$ & $5\left({ }^{b}\right)$ \\
\hline Has experienced symptoms in the past 12 months despite PsA medication, $n(\%)^{c}$ & $37(97)$ & $1237(97)$ \\
\hline \multicolumn{3}{|l|}{ Symptoms experienced ${ }^{\mathrm{d}}$} \\
\hline Musculoskeletal $^{\mathrm{c}}$ & $34(90)$ & $1155(91)$ \\
\hline Skin/nail ${ }^{\mathrm{c}}$ & $25(66)$ & $784(62)$ \\
\hline Unusual fatigue & $13(36)$ & $486(38)$ \\
\hline Would change something about current medication, $n(\%)^{\mathrm{c}}$ & $35(93)$ & $1183(93)$ \\
\hline \multicolumn{3}{|l|}{ Aspects to change about current PsA medication $(\mathrm{s}), n(\%)^{\mathrm{e}}$} \\
\hline Take fewer medications & $16(43)$ & $447(35)$ \\
\hline Efficacy of medications to relieve musculoskeletal symptoms & $16(42)$ & $503(40)$ \\
\hline Number or severity of side effects & $14(37)$ & $511(40)$ \\
\hline Ability to take medications orally versus parenterally & $13(34)$ & $404(32)$ \\
\hline Number of restrictions (e.g., can't drink alcohol, special diet, family planning) & $12(31)$ & $371(29)$ \\
\hline Efficacy of medications to relieve skin/nail symptoms & $11(30)$ & $393(31)$ \\
\hline Frequency of medication intake & $10(26)$ & $412(32)$ \\
\hline Cost or coverage availability from healthcare system & $9(23)$ & $367(29)$ \\
\hline Medication storage & $7(19)$ & $189(15)$ \\
\hline
\end{tabular}


Table 2 continued

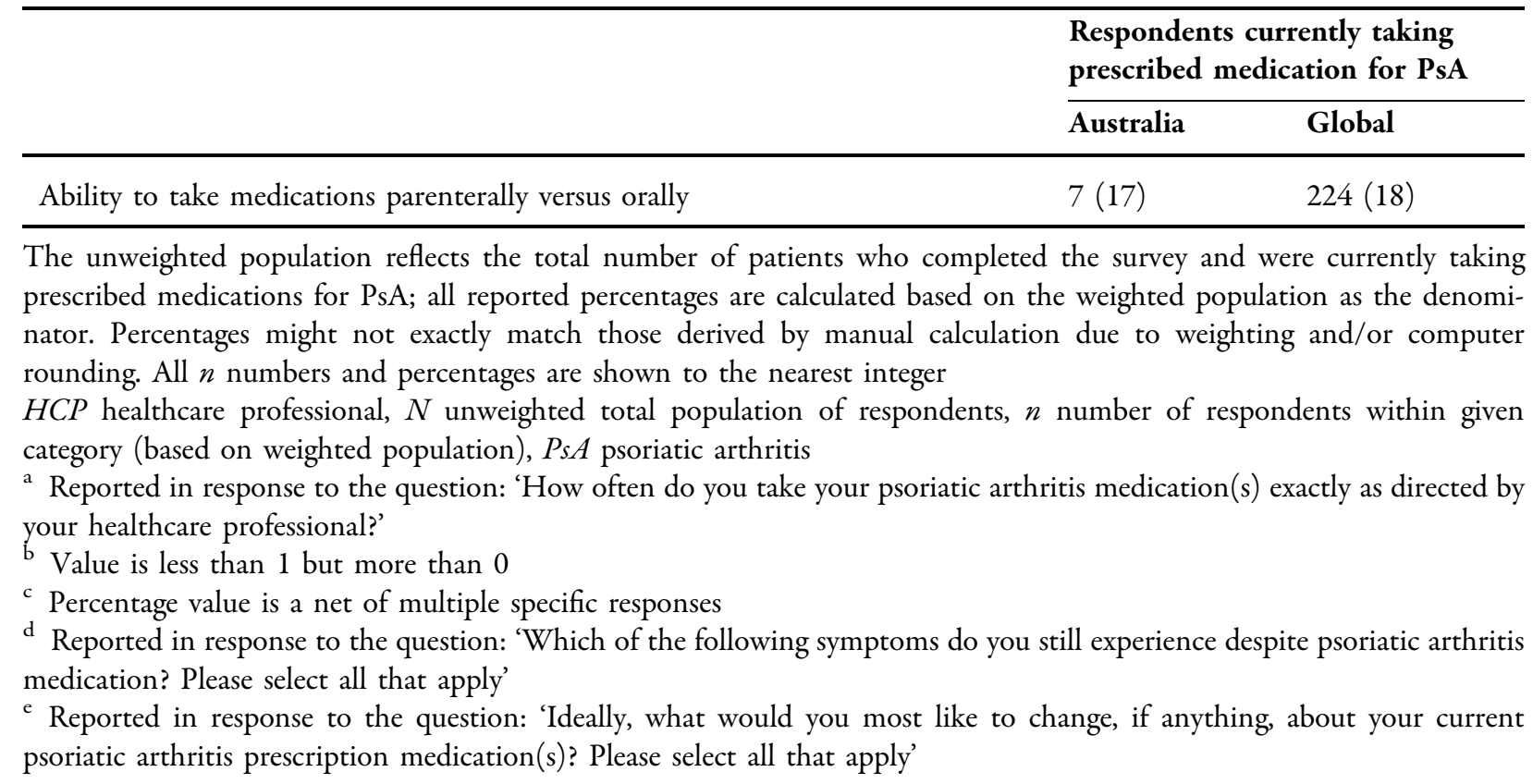

ensuring patients understand the next step in disease management, and working with the patient to make treatment decisions (Fig. 1).

The majority of patients in Australia wished that they talked more with their HCP regarding their PsA and treatment goals; however, approximately half of patients in Australia were concerned that asking too many questions would impact the quality of care that they received (Fig. 2a). Around half of patients in Australia reported that they often told their HCP that they were 'fine', but that they still experienced symptoms, suggesting that patient-HCP communication could be better (Fig. 2a). Most global patients wished for increased communication with their HCP relating to their disease and treatment goals, and around half were worried that asking too many questions would affect the quality of care that they received (Fig. $2 b$ ).

Most patients in Australia and most global patients who had visited a rheumatologist or dermatologist in the past 12 months reported discussing the following with their HCP: response/satisfaction with PsA treatment regimen, overall health, treatment goals, disease management and impact of PsA on physical activity (Fig. 3).
In Australia, $45 \%$ and $43 \%$ of patients with PsA responded with 'strongly agree' when asked if they felt comfortable raising concerns or fears with their rheumatologist and dermatologist, respectively. Similarly, approximately half of global patients with PsA responded with 'strongly agree' when asked if they felt comfortable raising concerns or fears with their HCP.

Among patients in Australia, commonly reported reasons for being uncomfortable with raising concerns with their HCP included: not feeling that there is much their HCP can control or change about their PsA management, deferring to HCP expertise, not having enough time with their HCP, not wanting to be seen as a difficult patient, and worrying that it will affect the quality of care received (see Fig. S1a in the electronic supplementary material). It should be noted that the number of patients in Australia who responded to this survey question regarding their dermatologist was very low $(n=21)$; the results should therefore be interpreted with caution.

Among global patients, frequently reported reasons for feeling uncomfortable discussing concerns with their HCP included: deferring to HCP expertise, not wanting to be seen as a 


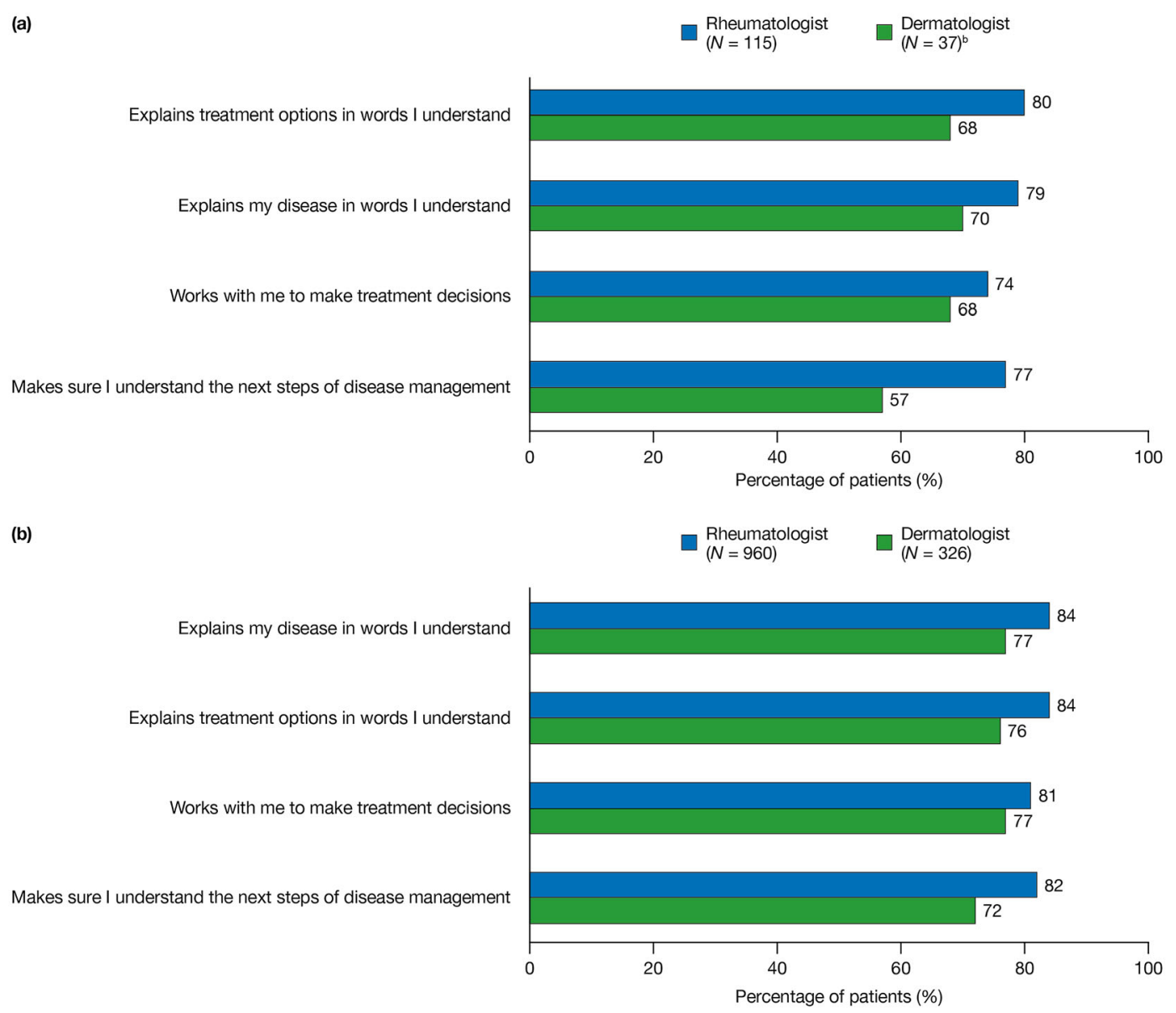

Fig. 1 Aspects of communication that patients treated by rheumatologists or dermatologists felt their HCP handled 'very/somewhat well'a, among a respondents in Australia with PsA and $\mathbf{b}$ global respondents with PsA. All reported percentages are calculated based on the weighted population as the denominator (Australia: rheumatologist-treated patients, $N=29 /$ dermatologist-treated patients, $N=9$; global: rheumatologist-treated patients, $N=983 /$ dermatologist-treated patients, $N=303$ ). Percentages might not exactly match those derived by manual calculation due to weighting and/or computer rounding. ${ }^{a}$ Reported in

difficult patient, and worrying about learning that their PsA medication is failing (see Fig. S1b in the electronic supplementary material).

\section{PsA Management Goals}

The most commonly reported PsA management goals according to patients in Australia included: increase level of physical activity (51\%), be response to the question: 'Thinking of your discussions with the HCP who is mostly responsible for managing your symptoms of PsA, what aspects do you feel they do well and what could they do better? Rheumatologist/dermatologist is mostly responsible for managing PsA'. Data represent patients reporting 'very/somewhat well'. 'Very small base size $(n<50)$; results should be interpreted as directional only. HCP healthcare professional, $N$ unweighted total population of respondents who considered their rheumatologist/dermatologist as the primary HCP for managing their PsA, Ps $A$ psoriatic arthritis

able to conduct daily activities more comfortably (50\%), prevent or slow further joint damage $(50 \%)$, and reduce joint swelling, tenderness and/or pain (50\%) (Table 3). For global patients, the most frequently reported goals for managing their PsA included: prevent or slow further joint damage (51\%), reduce joint swelling, tenderness and/or pain (50\%), and put PsA into remission (50\%) (Table 3). Overall, similar 
(a)

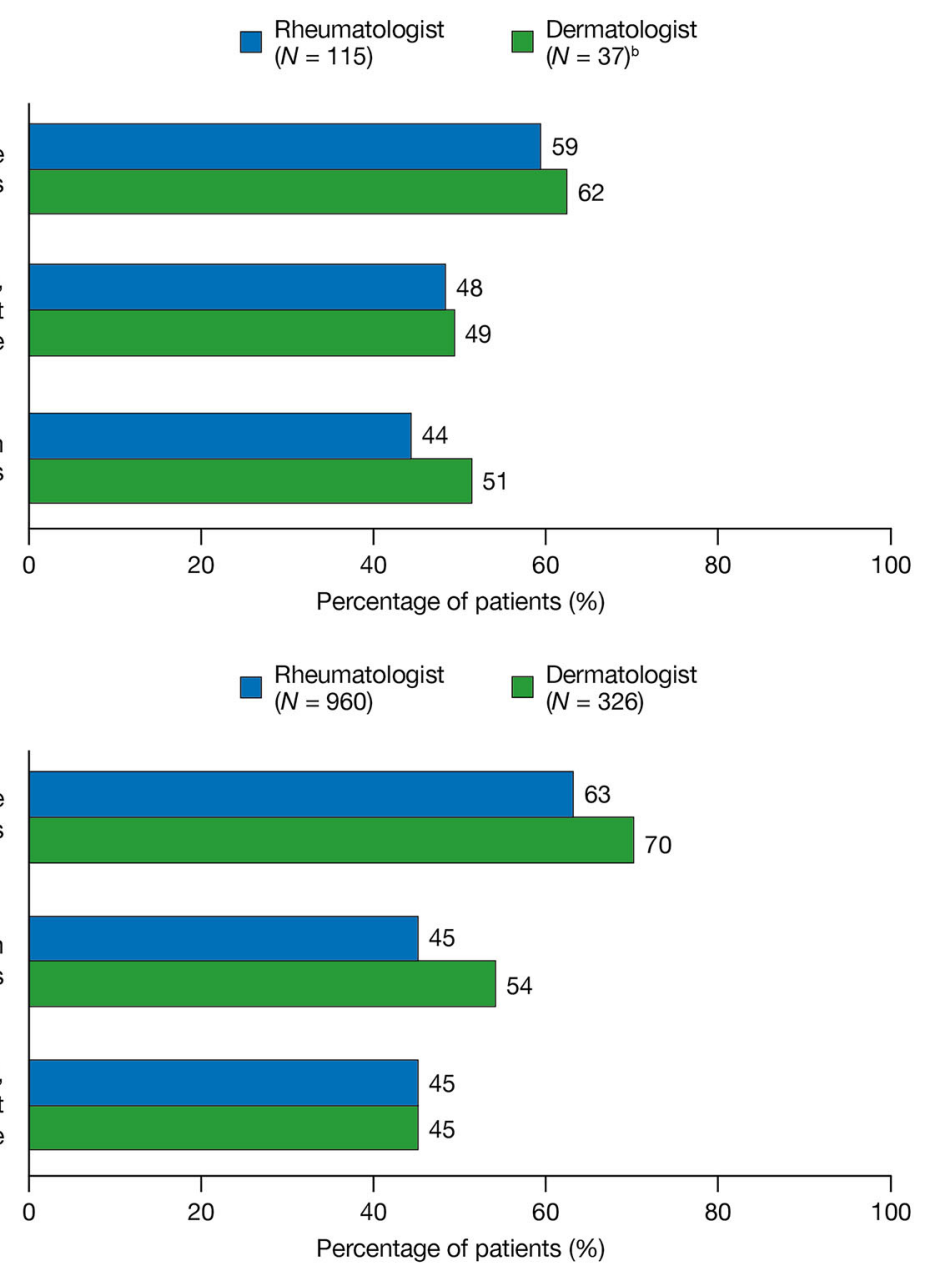

Fig. 2 Statements patients treated by rheumatologists or dermatologists 'strongly/somewhat' agreed with pertaining to HCP communication ${ }^{\mathrm{a}}$, among a respondents in Australia with PsA and $\mathbf{b}$ global respondents with PsA. All reported percentages are calculated based on the weighted population as the denominator (Australia: rheumatologist-treated patients, $N=29 /$ dermatologisttreated patients, $N=9$; global: rheumatologist-treated patients, $\quad N=983 /$ dermatologist-treated patients, $N=303$ ). Percentages might not exactly match those derived by manual calculation due to weighting and/or

computer rounding. ${ }^{\text {a }}$ Reported in response to the question: 'With respect to your communication with the HCP who is mostly responsible for managing your PsA, how much do you agree or disagree with each of the following statements?'. Data represent patients who responded that they 'strongly/somewhat' agreed. 'Very small base size $(n<50)$; results should be interpreted as directional only. $H C P$ healthcare professional, $N$ unweighted total population of respondents who considered their rheumatologist/dermatologist as the primary HCP for managing their PsA, Ps $A$ psoriatic arthritis

proportions of patients in Australia and global patients selected each goal.

\section{DISCUSSION}

Demographics for patients in Australia and global patients with PsA were generally similar,

although a higher proportion of global patients were taking biologic DMARDs at the time of the survey, compared with patients in Australia. Patient perceptions of PsA treatment and communication with their HCP were generally aligned between the Australian and global patient survey respondents. Most patients in Australia, and most global patients, were 
(a)

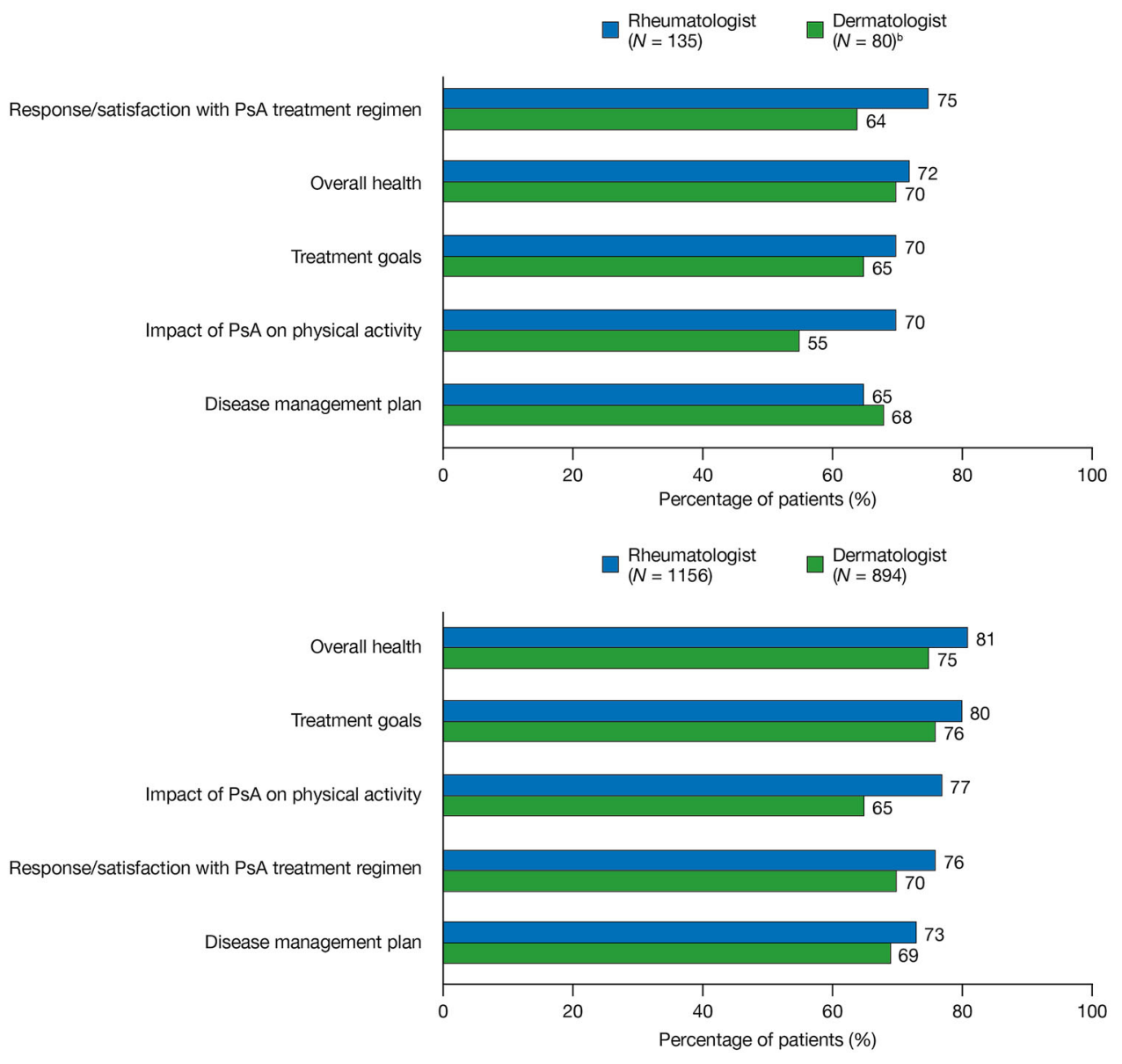

Fig. 3 Aspects of PsA management discussed with $\mathrm{HCP}^{\mathrm{a}}$ among a respondents in Australia with PsA and b global respondents with PsA, who had visited a rheumatologist or dermatologist in the past 12 months. All reported percentages are calculated based on the weighted population as the denominator (Australia: rheumatologist-treated patients, $N=34 /$ dermatologist-treated patients, $N=20$; global: rheumatologist-treated patients, $N=1172 /$ dermatologist-treated patients, $N=939$ ). Percentages might not exactly match those derived by manual calculation due to

satisfied with their PsA medication; however, the large majority still experienced symptoms, reported their overall health as poor or fair, and would change something about their medication. One of the most commonly desired medication changes was a reduction in the number of medications being taken, despite the recognized importance for prescribers to simplify patient drug regimens as much as possible to reduce unintentional non-adherence [14]. weighting and/or computer rounding. ${ }^{\mathrm{a}}$ Reported in response to the question: 'In the last 12 months, have you discussed/conducted each of the following with your rheumatologist/dermatologist regarding PsA?' Data represent patients who responded 'yes'. 'S Small base size $(n<100)$; results should be interpreted as directional only. $H C P$ healthcare professional, $N$ unweighted total population of respondents who had visited a rheumatologist/dermatologist in the last 12 months, $P s A$ psoriatic arthritis

Moreover, although most patients in Australia and most global patients were satisfied with the communication with their HCP, most wished that they could talk more with their HCP regarding their PsA and treatment goals. Strikingly, approximately half of all survey respondents felt that asking their HCP too many questions would impact the quality of care that they would receive. In addition, approximately half of patients in Australia and half of the 
Table 3 Patient-reported PsA management goals

\begin{tabular}{lll}
\hline & \multicolumn{2}{l}{ Respondents } \\
\cline { 2 - 3 } & Australia & Global \\
\hline Unweighted population, $N$ & 152 & 1286 \\
Weighted base, $N$ & 39 & 1286 \\
Patient-reported PsA management goals, $n(\%)^{\mathrm{a}}$ & & \\
Increase level of physical activity & & \\
Be able to conduct daily activities more comfortably & $20(51)$ & $588(46)$ \\
Prevent or slow further joint damage & $19(50)$ & $636(49)$ \\
Reduce joint swelling, tenderness and/or pain & $19(50)$ & $658(51)$ \\
Put PsA into remission (meaning my disease is controlled with few to no symptoms) & $19(50)$ & $645(50)$ \\
Reduce fatigue & $18(46)$ & $603(47)$ \\
Reduce stiffness & $17(45)$ & $610(47)$ \\
Worry less about PsA & $16(41)$ & $491(38)$ \\
Improve skin symptoms & $15(39)$ & $599(47)$ \\
Improve emotional well-being & $15(39)$ & $634(49)$ \\
Prevent or limit disability & $14(38)$ & $469(36)$ \\
\hline
\end{tabular}

The unweighted population reflects the total number of patients who completed the survey; all reported percentages are calculated based on the weighted population as the denominator. Percentages might not exactly match those derived by manual calculation due to weighting and/or computer rounding. All $n$ numbers and percentages are shown to the nearest integer

$N$ unweighted total population of respondents, $n$ number of respondents within given category (based on weighted population), PsA psoriatic arthritis

a Reported in response to the question: 'What are your goals for managing PsA? Please select all that apply'

global patients were not comfortable raising concerns or fears.

In a prospective international study of 410 patients with PsA that evaluated patient- and physician-perceived remission and LDA (measured by 'yes/no' questions), physicians appeared to consider patients to be in remission more often than patients did, whereas the opposite was true for LDA [15]. Similarly, a recent post hoc analysis of 223 patients with PsA showed disagreement between HCP-judged remission (measured by a 'yes/no' question), and patients considering themselves to be 'well' (measured by Patient Acceptable Symptom State, a single-question tool with potential for use as an alternative measure of disease activity in patients with PsA in clinical practice [16])
[17]. Additionally, a multicenter European study of 460 patients with PsA revealed discordance between HCP and patient global assessments of disease activity [18]. Given the mismatch between HCP and patient perceptions of PsA activity [19], it is perhaps unsurprising that about three in five patients with PsA in our survey wished that they could talk more to their HCP about their disease and treatment goals.

A 2017 working group of patient representatives and rheumatologists identified misalignment of treatment goals between HCPs and patients, where HCPs focused on achieving validated clinical measures, and patients focused on improving quality of life and retaining social and physical function [10]. The 
working group also highlighted that HCPs and patients may have different perceptions of the meaning of disease remission. Notably, some patients who are determined to be in a state of remission, LDA, or minimal disease activity can have residual disease activity, which may present as pain, functional impairment, joint tenderness, or other clinical manifestations [20]. Focusing on patients' treatment goals and enhanced patient-HCP communication were recommended by the working group for improving clinical outcomes for patients with PsA [10]. Intriguingly, a survey of 435 participants in Australia with osteoarthritis revealed that, amongst other factors, the effectiveness of communication between patients and HCPs was a significant predictor of overall satisfaction with HCP care [21].

Patients are more likely to adhere to their medication regimen if they share their HCPs' beliefs regarding health outcomes [22]. A recent focus group study, including patients in Australia with gout, osteoporosis and rheumatoid arthritis, and their caregivers, identified that trust in the physician, physician's knowledge, and medication properties (e.g., effectiveness and side effects) were important factors contributing to medication adherence. The authors of the study highlighted that increased trust and knowledge exchange between doctors and patients could improve medication adherence [23]. A focus group study including 30 inpatients from four Australian hospitals confirmed that staff-patient communication (and communication between staff members), and the sharing of high-quality information with patients and their families or carers, are important for patient satisfaction [24].

An Australian government-supported study on patient perception of DMARD use in patients with rheumatoid arthritis $(N=1149)$ and spondyloarthritis $(N=191)$ identified key barriers to medication adherence, most of which stemmed from inadequate sharing of highquality information, leading to patient apprehension towards their disease management strategy [25]. It is important to note that, in Australia, the majority of patients with PsA are managed in private community practices, and this is where the bulk of biologic DMARD prescribing occurs. While the majority of patients with psoriasis are also treated in private and community practices, most biologic DMARD use in psoriasis is managed in public hospital dermatology clinics. With this in mind, a more targeted approach to improve patient-HCP communication and information sharing in these settings should be prioritized.

A limitation of this analysis was the relatively small number of patients in Australia who responded to the survey overall (and to certain survey questions in particular), with a base sample size of only 152; the findings should therefore be interpreted with care. Additionally, as the data from patients in Australia were included in the overall global respondent pool, no direct statistical comparisons could be made between Australian versus global results. It should be noted that only eight countries were included in the global cohort; ideally, additional countries would need to be incorporated for a more accurate representation of the global PsA patient population. Finally, this study relied on self-reporting from patients with PsA; therefore, we cannot verify the accuracy of their responses to each of the questions asked.

\section{CONCLUSIONS}

This study of patients in Australia with PsA highlights an apparent paradox, with high rates of patient-reported satisfaction with their disease management juxtaposed with substantial rates of self-reported poor or fair overall current health, persistent symptoms, the desire to change aspects of their PsA management, and concerns about communication with their HCP. Our results support the need for a more collaborative approach between HCPs and patients regarding PsA management.

\section{ACKNOWLEDGMENTS}

Funding. This study was funded by Pfizer Inc. The journal's Rapid Service Fee for this article was also funded by Pfizer Inc. 
Medical Writing Assistance. Medical writing support, under the guidance of the authors, was provided by Dominic Singson, MD, and Emma Mitchell, PhD, CMC Connect, McCann Health Medical Communications and was funded by Pfizer Inc, New York, NY, USA in accordance with Good Publication Practice (GPP3) guidelines (Ann Intern Med. 2015;163:461-4).

Authorship. All named authors meet the International Committee of Medical Journal Editors (ICMJE) criteria for authorship for this article, take responsibility for the integrity of the work as a whole, and have given their approval for this version to be published.

Disclosures. Irwin Lim has received honoraria from, and been involved in advisory boards for, AbbVie, Janssen, Novartis, Pfizer Inc, Roche, and UCB. Pascal Richette has received honoraria and/or research funding from AbbVie, Celgene, Janssen, Lilly, Novartis, Pfizer Inc, Roche, and UCB. Ruben Queiro-Silva has received honoraria and/or research funding from AbbVie, Celgene, Janssen, Lilly, MSD, Novartis, Pfizer Inc, and UCB. Jade Moser is an employee of Harris Insights \& Analytics. Joseph C. Cappelleri, Ho Yin Ng, and David Witcombe are employees and shareholders of Pfizer Inc.

\section{Compliance with Ethics Guideli-} nes. Patients were recruited from online market research panels made up of members who agreed to participate in this type of research. Qualified respondents provided informed consent to complete the research. The surveys were non-interventional and were not conducted as a clinical study. All respondents agreed to participate, however, due to the nature of the study, ethics approval was not required.

Data Availability. Upon request, and subject to certain criteria, conditions and exceptions (see https://www.pfizer.com/science/ clinical-trials/trial-data-and-results for more information), Pfizer will provide access to individual de-identified participant data from Pfizersponsored global interventional clinical studies conducted for medicines, vaccines, and medical devices (1) for indications that have been approved in the US and/or EU, or (2) in programs that have been terminated (i.e., development for all indications has been discontinued). Pfizer will also consider requests for the protocol, data dictionary, and statistical analysis plan. Data may be requested from Pfizer trials 24 months after study completion. The deidentified participant data will be made available to researchers whose proposals meet the research criteria and other conditions, and for which an exception does not apply, via a secure portal. To gain access, data requestors must enter into a data access agreement with Pfizer.

Open Access. This article is licensed under a Creative Commons Attribution-NonCommercial 4.0 International License, which permits any non-commercial use, sharing, adaptation, distribution and reproduction in any medium or format, as long as you give appropriate credit to the original author(s) and the source, provide a link to the Creative Commons licence, and indicate if changes were made. The images or other third party material in this article are included in the article's Creative Commons licence, unless indicated otherwise in a credit line to the material. If material is not included in the article's Creative Commons licence and your intended use is not permitted by statutory regulation or exceeds the permitted use, you will need to obtain permission directly from the copyright holder. To view a copy of this licence, visit http://creativecommons.org/licenses/by$\mathrm{nc} / 4.0 /$.

\section{REFERENCES}

1. Gladman DD, Antoni C, Mease P, Clegg DO, Nash P. Psoriatic arthritis: epidemiology, clinical features, course, and outcome. Ann Rheum Dis. 2005;64: ii14-7.

2. Coates LC, Kavanaugh A, Mease PJ, et al. Group for Research and Assessment of Psoriasis and Psoriatic Arthritis 2015 treatment recommendations for psoriatic arthritis. Arthritis Rheumatol. 2016;68: 1060-71.

3. Alinaghi F, Calov M, Kristensen LE, et al. Prevalence of psoriatic arthritis in patients with psoriasis: a 
systematic review and meta-analysis of observational and clinical studies. J Am Acad Dermatol. 2019;80:251-65.e19.

4. Rosen CF, Mussani F, Chandran V, Eder L, Thavaneswaran A, Gladman DD. Patients with psoriatic arthritis have worse quality of life than those with psoriasis alone. Rheumatology. 2012;51:571-6.

5. Husted JA, Gladman DD, Farewell VT, Cook RJ. Health-related quality of life of patients with psoriatic arthritis: a comparison with patients with rheumatoid arthritis. Arthritis Care Res. 2001;45: 151-8.

6. Husted JA, Thavaneswaran A, Chandran V, et al. Cardiovascular and other comorbidities in patients with psoriatic arthritis: a comparison with patients with psoriasis. Arthritis Care Res. 2011;63:1729-35.

7. Mease PJ, Armstrong AW. Managing patients with psoriatic disease: the diagnosis and pharmacologic treatment of psoriatic arthritis in patients with psoriasis. Drugs. 2014;74:423-41.

8. Liu J-T, Yeh H-M, Liu S-Y, Chen K-T. Psoriatic arthritis: epidemiology, diagnosis, and treatment. World J Orthop. 2014;5:537-43.

9. Machado PM, Raychaudhuri SP. Disease activity measurements and monitoring in psoriatic arthritis and axial spondyloarthritis. Best Pract Res Clin Rheumatol. 2014;28:711-28.

10. Garrido-Cumbrera M, Hillmann O, Mahapatra R, et al. Improving the management of psoriatic arthritis and axial spondyloarthritis: roundtable discussions with healthcare professionals and patients. Rheumatol Ther. 2017;4:219-31.

11. Smolen JS, Schöls M, Braun J, et al. Treating axial spondyloarthritis and peripheral spondyloarthritis, especially psoriatic arthritis, to target: 2017 update of recommendations by an international task force. Ann Rheum Dis. 2018;77:3-17.

12. Coates LC, Orbai A-M, Azevedo VF, et al. Results of a global, patient-based survey assessing the impact of psoriatic arthritis discussed in the context of the Psoriatic Arthritis Impact of Disease (PsAID) questionnaire. Health Qual Life Outcomes. 2020;18:173.

13. Kish L. Survey sampling. New York: John Wiley \& Sons; 1965.

14. Usherwood T. Encouraging adherence to long-term medication. Aust Prescr. 2017;40:147-50.
15. Gorlier C, Orbai A-M, Puyraimond-Zemmour D, et al. Comparing patient-perceived and physicianperceived remission and low disease activity in psoriatic arthritis: an analysis of 410 patients from 14 countries. Ann Rheum Dis. 2019;78:201-8.

16. Lubrano E, Scriffignano S, Azuaga AB, Ramirez J, Canete JD, Perrotta FM. Assessment of the Patient Acceptable Symptom State (PASS) in psoriatic arthritis: association with disease activity and quality of life indices. RMD Open. 2020;6:e001170.

17. Queiro R. Mismatch between the impact of disease perceived by patients and the state of clinical remission assessed by physicians in psoriatic arthritis. Clin Exp Rheumatol. 2020;38:333-6.

18. Desthieux C, Granger B, Balanescu AR, et al. Determinants of patient-physician discordance in global assessment in psoriatic arthritis: a multicenter European study. Arthritis Care Res. 2017;69:1606-11.

19. Queiro R. Remission and stringent treatment goals in psoriatic arthritis: doctors' opinion is not enough. Joint Bone Spine. 2019;86:269-70.

20. Lubrano E, Scriffignano S, Perrotta FM. Residual disease activity and associated factors in psoriatic arthritis. J Rheumatol. 2020;47:1490-5.

21. Basedow M, Hibbert P, Hooper T, Runciman W, Esterman A. Australians with osteoarthritis: satisfaction with health care providers and the perceived helpfulness of treatments and information sources. J Multidiscip Healthc. 2016;9:387-94.

22. Christensen AJ, Howren MB, Hillis SL, et al. Patient and physician beliefs about control over health: association of symmetrical beliefs with medication regimen adherence. J Gen Intern Med. 2010;25: 397-402.

23. Kelly A, Tymms K, de Wit M, et al. Patient and caregiver priorities for medication adherence in gout, osteoporosis and rheumatoid arthritis: nominal group technique. Arthritis Care Res. 2020;72:1410-9.

24. Rapport F, Hibbert P, Baysari M, et al. What do patients really want? An in-depth examination of patient experience in four Australian hospitals. BMC Health Serv Res. 2019;19:38.

25. Kelly A, Tymms K, Tunnicliffe DJ, et al. Patients' attitudes and experiences of disease-modifying antirheumatic drugs in rheumatoid arthritis and spondyloarthritis: a qualitative synthesis. Arthritis Care Res. 2018;70:525-32. 